\title{
PRIMENESS OF THE ENVELOPING ALGEBRA OF HAMILTONIAN SUPERALGEBRAS
}

\author{
MARK C. WILSON
}

In 1990 Allen Bell presented a sufficient condition for the primeness of the universal enveloping algebra of a Lie superalgebra. Let $Q$ be a nonsingular bilinear form on a finite-dimensional vector space over a field of characteristic zero. In this paper we show that Bell's criterion applies to the Hamiltonian Cartan type superalgebras determined by $Q$, and hence that their enveloping algebras are semiprimitive.

\section{INTRODUCTION}

Let $L=L_{+}+L_{-}$be a finite-dimensional Lie superalgebra over a field of characteristic zero, and let $U(L)$ be its universal associative enveloping (super)algebra. In [1] Bell gave the following simple criterion for primeness of $U(L)$. Let $\left\{f_{1}, \ldots, f_{n}\right\}$ be a basis for the odd part $L_{-}$of $L$. Form the product matrix $M=\left(\left[f_{i}, f_{j}\right]\right)$, considered as a matrix over the symmetric algebra $S\left(L_{+}\right)$. If $\operatorname{det} M \neq 0$ then $U(L)$ is prime.

Note that since $U(L)$ is a Jacobson ring (see for example [5]), if $U(L)$ is prime then it is also semiprimitive. As far as is known these last two properties may be equivalent for rings of the form $U(L)$.

The primeness question for enveloping algebras of the classical simple Lie superalgebras has been settled completely in [1] and [3]. An investigation into the applicability of Bell's criterion to the Cartan type Lie superalgebras was begun in [8], continued in [10] and is concluded in this paper and $[\mathbf{9}]$.

Here it is shown that the Hamiltonian algebras $H(Q)$ and $\widetilde{H}(Q)$ satisfy Bell's criterion. This immediately gives

THEOREM. Let $K$ be a field of characteristic zero, let $n \geqslant 4$ and let $Q$ be a nonsingular bilinear form on a $K$-vector space of dimension $n$. Then $U(H(Q))$ and $U(\tilde{H}(Q))$ are prime.

As a consequence of the results of the above-mentioned papers we have the following theorem.

THEOREM. Let $L$ be a finite-dimensional simple Lie superalgebra over an algebraically closed field of characteristic zero. Then $L$ satisfies Bell's criterion, and hence

Received 24th February, 1997

Copyright Clearance Centre, Inc. Serial-fee code: 0004-9729/97 \$A2.00+0.00. 
$U(L)$ is prime, unless $L$ is of one of the types: $b(n)$ for $n \geqslant 3 ; W(n)$ for odd $n \geqslant 5 ; S(n)$ for odd $n \geqslant 3$.

\section{The Hamiltonian superalgebras}

Good references for basic facts about Lie superalgebras are [2] and [7].

Let $K$ be a field of characteristic zero, $n$ a positive integer and $V$ an $n$-dimensional $K$-vector space. Let $\Lambda=\Lambda(V)$ be the Grassmann algebra of $V$. Recall that $\Lambda$ is an associative $\mathbb{Z}$-graded superalgebra. Fix a basis $\left\{v_{1}, \ldots, v_{n}\right\}$ for $V$. For each ordered subset $I=\left\{i_{1}, i_{2}, \ldots, i_{r}\right\}$ of $N=\{1,2, \ldots, n\}$ with $i_{1}<i_{2}<\cdots<i_{r}$, let $v_{I}$ be the product $v_{i_{1}} v_{i_{2}} \cdots v_{i_{r}}$. The set of all such $v_{I}$ forms a basis for $\Lambda$, where we interpret $1=v_{\emptyset}$ as the empty product, and the homogeneous component $\Lambda_{r}$ is spanned by the $v_{I}$ with $|I|=r$. The anticommutativity of multiplication in $\Lambda$ implies that

$$
v_{I} v_{J}= \begin{cases} \pm v_{I \cup J} & \text { if } I \cap J=\emptyset \\ 0 & \text { if } I \cap J \neq \emptyset\end{cases}
$$

The algebra $W=W(V)$ is the $\mathbb{Z}$-graded Lie superalgebra consisting of all superderivations of $\Lambda$. Every element of $W$ maps $V$ into $\Lambda$ and since it is a superderivation it is completely determined by its action on the generating subspace $V$. It follows that $W$ can be identified with $\Lambda \otimes_{K} V^{*}$ and we shall henceforth do so.

Under this identification the map $\partial_{i}=\partial / \partial_{v_{i}}$ corresponds to the dual of $v_{i}$ which we shall also denote $\partial_{i}$. The set of all $v_{I} \otimes \partial_{i}$ is then a homogeneous basis for $W$, the degree of such an element being equal to $|I|-1$.

For each symmetric bilinear form $Q$ on $V$ there are subalgebras of $W$ denoted by $H(Q)$ and $\widetilde{H}(Q)$. Their (rather complicated) definition can be found in [7, p.194] or [2, section 3.3.2]. If we extend $K$ to its algebraic closure then all such algebras become isomorphic to the algebra $\widetilde{H}(n)$ (respectively $H(n)$ ) defined below. Since Bell's criterion holds over a given field if and only if it holds over the algebraic closure of that field, it is sufficient to verify the criterion for the algebras $\widetilde{H}(n)$ and $H(n)$.

We now recall some basic facts about the Hamiltonian superalgebras. The subspace of $W$ spanned by all superderivations of the form

$$
D_{\lambda}=\sum_{i \in N} \partial_{i}(\lambda) \otimes \partial_{i}
$$

where $\lambda \in \Lambda$, is a Lie superalgebra called $\widetilde{H}=\widetilde{H}(n) . \widetilde{H}$ inherits a natural $\mathbb{Z}$-grading from $W$ and we have

$$
\widetilde{H}=\bigoplus_{r=-1}^{n-2} H_{r}
$$


The subalgebra $H=H(n)=\bigoplus_{r=-1}^{n-3} H_{r}=[\widetilde{H}, \widetilde{H}]$ is a simple Lie superalgebra of Cartan type.

The homogeneous component $H_{r}$ is isomorphic as a vector space (in fact as an $H_{0^{-}}$ module) to $\Lambda_{r+2}$ via $D_{\lambda} \mapsto \lambda$. Thus the superderivations $x_{I}=D_{v_{I}}$, where $\emptyset \neq I \subseteq N$, form a basis for $\widetilde{H}$, and $\operatorname{dim} H_{r}=\left(\begin{array}{c}n \\ r+2\end{array}\right)$.

\section{Computation}

It is known that the multiplication in $H$ satisfies

$$
\left[D_{\lambda}, D_{\mu}\right]= \pm D_{\{\lambda, \mu\}}
$$

where $\{\lambda, \mu\}=\sum_{i} \partial_{i}(\lambda) \partial_{i}(\mu)$. Note that this differs slightly from the notation in [2], and that the exact multiplication formula is not needed for our purposes.

It follows from (1) that $\partial_{i}\left(v_{I}\right) \partial_{i}\left(v_{J}\right)=0$ unless $I \cap J=\{i\}$, whence

$$
\left[x_{I}, x_{J}\right]= \begin{cases} \pm x_{I \Delta J} & \text { if }|I \cap J|=1 \\ 0 & \text { otherwise }\end{cases}
$$

where $\Delta$ denotes the symmetric difference (Boolean sum). Since $\Delta$ is the addition in the usual Boolean ring structure on the power set of $N$, this implies that for a given $A, I \subseteq N$, the equation $\left[x_{I}, x_{J}\right]= \pm x_{A}$ has at most one solution for $J$. This solution exists precisely when $A \Delta I \neq \emptyset$, that is when $I \nsubseteq A$ and $A \nsubseteq I$. Furthermore if $|I|$ is odd and $|A|$ even then $|J|=|A|+|I|-2|I \cap A|$ is necessarily odd. Thus every even $x_{A}$ appears (perhaps with a minus sign) in the product matrix, and each such $x_{A}$ appears at most once in each row or column.

\section{1. $n$ EVEN}

TheOREM 3.1. Let $n \geqslant 4$ be an even integer. Then $H(n)$ and $\widetilde{H}(n)$ satisfy Bell's criterion.

Proof: Write $n=2 m$. The highest odd degree occurring in $\widetilde{H}$ and $H$ is $n-3$. It follows that if we group the basis elements $x_{I}$ by increasing degree, then the product matrices for both $H$ and $\widetilde{H}$ are the same and that this common matrix has the block reverse triangular structure

$$
\left(\begin{array}{ccccc}
H_{-1,-1} & H_{-1,1} & \ldots & \ldots & H_{-1, n-3} \\
H_{1,-1} & H_{1,1} & \ldots & H_{1, n-5} & 0 \\
\vdots & \vdots & \vdots & \vdots & \vdots \\
H_{n-3,-1} & 0 & \ldots & 0 & 0
\end{array}\right)
$$

where $H_{\tau, s}$ is the block formed by the products of elements of degree $r$ with those of degree $s$. Furthermore each block on the reverse diagonal is square, since if $r+s=n-4$ 
then $\operatorname{dim} H_{r}=\left(\begin{array}{c}n \\ r+2\end{array}\right)=\left(\begin{array}{c}n \\ s+2\end{array}\right)=\operatorname{dim} H_{s}$. The product matrix is nonsingular if and only if each of these blocks is nonsingular.

Fix such a reverse diagonal block $H_{r, s}$ corresponding to products of elements of degree $r$ by those of degree $s=n-4-r$. Using the identification of $H_{n-4}$ with $\Lambda_{n-2}$ we can index the basis elements of $H_{n-4}$ by their (ordered) 2-element complements, for example $y_{13}=x_{N \backslash\{1,3\}}$. We now make the specialisation which sends $y_{i j}$ to 0 unless $j-i=m$, and call the $m$ remaining variables $z_{1}=y_{11^{\prime}}, \ldots, z_{m}=y_{m m^{\prime}}$. For each $i$ let $i^{\prime}=i+m(\bmod n)$. Note that $\left(i^{\prime}\right)^{\prime}=i$ and $z_{i}=z_{i^{\prime}}$. The image $B$ of the block $H_{r, s}$ under this specialisation is a matrix whose only possibilities for nonzero entries are $\pm z_{i}$ for some $i$.

We shall obtain a further block decomposition of $B$. By replacing all nonzero elements of $B$ by 1 's, we obtain a $(0,1)$ matrix which is the adjacency matrix of a unique graph $G=G(B)$. In other words, $G$ has vertices the $x_{I}$ and an edge joining $x_{I}$ and $x_{J}$ if and only if the product $\left[x_{I}, x_{J}\right]$ remains nonzero under our specialisation above. If for simplicity we label the vertex corresponding to $x_{I}$ by $I$, there is an edge in $G$ joining $I$ to $J$ if and only if $\left[x_{I}, x_{J}\right]= \pm z_{i}$ for some $i$. We shall say that in this case $I$ and $J$ are joined by an edge of colour $i$.

Finding a block decomposition of $B$ is equivalent to decomposing $G$ into disjoint subgraphs, which we now proceed to do. Fix $i \in N$. We determine exact conditions on $I$ and $J$ for there to exist an edge of colour $i$ joining them. It follows from (2) that this occurs if and only if either $I \cap J=\{i\}$ and $I \cup J=N \backslash\left\{i^{\prime}\right\}$, or $I \cap J=\left\{i^{\prime}\right\}$ and $I \cup J=N \backslash\{i\}$. Thus there is an edge of colour $i$ joining $I$ and $J$ if and only if $|I|+|J|=n$, one of $i$ or $i^{\prime}$ belongs to both $I$ and $J$ and the other belongs to neither. Furthermore, for a given $I \neq N$, there is at most one edge of a given colour at the vertex $I$. Also there is at least one edge of some colour at the vertex $I$ : since $I \neq N$, for some $i$ we must have $i \in I$ and $i^{\prime} \notin I$.

We now obtain a further block decomposition of $B$ by showing that the set of colours occurring at a given vertex of $G(B)$ is constant on each component. To this end, we first show that vertices distance 2 apart have the same colours. Suppose that $I$ and $J$ are linked by an edge of colour $i$. Then without loss of generality $I \cap J=\{i\}$ and $I \cup J=N \backslash\left\{i^{\prime}\right\}$. Let $K$ be linked to $J$. If $J$ and $K$ are linked by an edge of colour $j$ then either $\left\{i, i^{\prime}\right\}=\left\{j, j^{\prime}\right\}$, in which case $K=I$, or $\left\{i, i^{\prime}\right\} \cap\left\{j, j^{\prime}\right\}=\emptyset$. In the latter case we can assume $J \cap K=\{j\}$ and $J \cup K=N \backslash\left\{j^{\prime}\right\}$. Thus $i^{\prime} \in K$ since $i^{\prime} \in J \cup K$ but $i^{\prime} \notin J$. Let $X=J \cup\left\{i^{\prime}, j^{\prime}\right\} \backslash\{i, j\}$. Then $|X|=|J|, K \cap X=\left\{i^{\prime}\right\}, K \cup X=N \backslash\{i\}$ and so $K$ and $X$ are linked by an edge of colour $i$. Thus every colour occurring at $I$ also occurs at $K$, and by symmetry $I$ and $K$ have the same colours.

It follows that if $I$ and $J$ are joined by an edge then they have the same colours, since if an edge of some colour $i$ joins $I$ and $L$, then $J$ and $L$ have the same colours by above and so the colour $i$ occurs at $J$. By induction on the length of a path joining 
two vertices, the set of colours occurring at a vertex is constant on components. This decomposes $G(B)$ into a union of disjoint subgraphs, each corresponding to a given set of colours. Hence $B$ decomposes as a direct sum of smaller blocks, each of which is parametrised by some nonempty subset of the set of colours.

Now fix such a block corresponding to a given set of colours. This matrix is such that in every row and column, each variable which is present occurs exactly once, perhaps with a minus sign. Then by specialising all but one of these variables to zero we obtain a nonsingular monomial matrix. This shows that the original product matrix for $H(n)$ and $\widetilde{H}(n)$ is nonsingular.

The fact that the Noetherian rings $R=U(H)$ and $S=U(\widetilde{H})$ are simultaneously prime is not a surprise. The component $H_{n-2}$ is 1-dimensional, spanned by $x$ say. Since $[x, H] \subseteq[\widetilde{H}, \widetilde{H}]=H$, ad $x$ stabilises $R$. When $n$ is even then ad $x$ is an ordinary derivation and so $S$ is the differential polynomial ring $R[x ; \operatorname{ad} x]$. It is a well-known fact (see for example [6, Proposition 8.3.32]) that in this situation $R$ is prime if and only if $S$ is.

3.2. $n$ ODD This case reduces rather easily to the previous one.

TheOREM 3.2. Let $n \geqslant 5$ be odd. Then $H(n)$ and $\widetilde{H}(n)$ satisfy Bell's criterion.

Proof: Let $M, \widetilde{M}$ be the product matrices for $H(n), \widetilde{H}(n)$ respectively. The top degree $n-2$ occurring in $\widetilde{H}(n)$ is odd, and $\operatorname{dim} H_{n-2}=1$. Thus $\widetilde{M}$ is obtained from $M$ by adding another row and column. Since this procedure either leaves the rank unchanged or increases the rank by 1 , it suffices to show that $\widetilde{M}$ is nonsingular.

We decompose $\widetilde{M}$ into 4 blocks as follows. Group the rows indexed by those $I$ for which $n \in I$ together and follow them by the rows for which $n \notin I$. Do the same for the columns. This gives an obvious $2 \times 2$ block structure. Make the specialisation which sets all even $x_{I}$ with $n \in I$ to zero. Then $\widetilde{M}$ specialises to a matrix of the form $\left(\begin{array}{ll}x & 0 \\ 0 & Y\end{array}\right)$. It suffices to show that $X$ and $Y$ are nonsingular.

The matrix $Y$ has entries which are the pairwise products of the $x_{I}$ with $I \subseteq$ $\{1, \ldots, n-1\}$ and hence is just a product matrix for $H(n-1)$. Thus $Y$ is nonsingular by Theorem 3.1.

Now choose $I$ with $n \in I$. Since $I \neq N$, both $I \nsubseteq N \backslash\{n\}$ and $N \backslash\{n\} \nsubseteq I$ hold and so there is precisely one $J$ with $n \in J$ for which $\left[x_{I}, x_{J}\right]= \pm x_{N \backslash\{n\}}$. Thus in $X$ every row and column has precisely one occurrence of $\pm x_{N \backslash\{n\}}$, so specialising to zero all variables except this one yields a nonsingular monomial matrix.

It is not as obvious a priori that the rings $R=U(H)$ and $S=U(\widetilde{H})$ should be simultaneously prime. Let $x$ span $H_{n-2}$. Then $[x, x]=2 x^{2}=0$ and so $S=R[x ; \delta] / I$, where $\delta$ is the skew derivation ad $x$ and $I$ is the ideal generated by $x^{2}$. Obviously $S$ prime implies $R$ prime but the converse for extensions of this type (see [4]) requires extra hypotheses regarding the action of $\delta$ on the symmetric Martindale quotient ring of $R$ which seem 
difficult to verify in our situation.

\section{REFERENCES}

[1] A.D. Bell, 'A criterion for primeness of enveloping algebras of Lie superalgebras', J. Pure Appl. Algebra 69 (1990), 111-120.

[2] V.G. Kac, 'Lie superalgebras', Adv. in Math. 26 (1977), 8-96.

[3] E. Kirkman and J. Kuzmanovich, 'Minimal prime ideals in enveloping algebras of Lie superalgebras', Proc. Amer. Math. Soc. 124 (1996), 1693-1702.

[4] A. Milinski, 'Actions of pointed Hopf algebras on prime algebras', Comm. Algebra 23 (1995), 313-333.

[5] I.M. Musson, 'Enveloping algebras of Lie superalgebras: a survey', Contemp. Math. 124 (1992), 141-149.

[6] L. Rowen, Ring theory (Academic Press, Boston, 1988).

[7] M. Scheunert, The theory of Lie superalgebras, Lecture Notes in Mathematics 716, (Springer-Verlag, Berlin, Heidelberg, New York, 1979).

[8] M.C. Wilson, 'Primeness of the enveloping algebras of a Cartan type Lie algebra', Proc. Amer. Math. Soc. 124 (1996), 383-387.

[9] M.C. Wilson and G. Pritchard, 'Primeness of the enveloping algebra of the special Lie superalgebras', Arch. Math. (Basel) (to appear).

[10] M.C. Wilson, G. Pritchard and D.H. Wood, 'Bell's primeness criterion for $W(2 n+1)$ ', Experiment. Math. (to appear).

Department of Mathematics

University of Auckland

Private Bag 92019 Auckland

New Zealand

e-mail: wilson@math.auckland.ac.nz 